\title{
Appendix: Reciprocity in Groups and the Limits to Social Capital.
}

Francis Bloch, Garance Genicot and Debraj Ray.

\section{Existence of Equilibrium Values.}

Proposition 1. An equilibrium exists.

Proof . Fix any value $v$ in some large compact interval $V$ containing all feasible payoffs. For any state $\omega$ and group $S$ of size $s$, define

$$
\Delta(\omega, v, S)=\max \left\{\min _{i}\left\{(1-\delta) c\left(q, \alpha_{i}\right)-\delta\left[v-v^{*}(s)\right]\right\}, 0\right\} .
$$

Obviously, $\Delta(\omega, v, S)>0$ if and only if $S$ has a strictly profitable deviation under $v$.

Define a collection $\phi^{1}(\omega, v)$ of symmetric probability distributions on $(\omega, v)$ by:

$[i]$ the singleton set containing

$$
q(\omega)=0 \text { and } p(\omega, v, S)=\frac{\Delta(\omega, v, S)}{\sum_{T} \Delta(\omega, v, T)} \forall S
$$

if some strictly profitable deviation exists at $(\omega, v)$, and

[ii] the collection of all symmetric probability distributions of the form $(p, q)$ otherwise, under the restriction that $p(\omega, v, S)$ can have positive value only if $S$ has a weakly profitable deviation.

Lemma 1. For each $\omega, \phi^{1}(\omega, v)$ is nonempty, convex-valued, and upperhemi-continuous (uhc) in $v$.

Proof. Obviously, $\phi^{1}(\omega, v)$ is nonempty and convex-valued for each $(\omega, v)$. Now we claim that it is uhc in $v$. To this end, let $v^{k}$ be some sequence in $V$ converging to $v$. Consider a corresponding sequence $\left(p^{k}, q^{k}\right) \in \phi^{1}\left(\omega, v^{k}\right)$ (we omit the explicit dependence on $\omega$ and $S$ for notational ease) and extract a convergent subsequence converging to 
some $(p, q)$ (but retain the original sequence notation). We claim that $(p, q) \in \phi^{1}(\omega, v)$.

This claim is obviously true if no strictly profitable deviation exists at $(\omega, v) .{ }^{1}$ So suppose that a strictly profitable deviation does exist at $(\omega, v)$. But then a strictly profitable deviation must exist for $k$ large enough, so that far enough out in the sequence, $\left(p^{k}, q^{k}\right)$ must be uniquely pinned down by the condition (1). Because $\Delta(\omega, v, S)$ is obviously continuous in $v$, we must have that $\left(p^{k}, q^{k}\right) \rightarrow(p, q)$ in this case as well.

We now construct a second map - this time, a function - that links symmetric probability systems to values. In line with condition [3] of an equilibrium (Consistent Values), simply define it by

$$
\phi^{2}(p, q)=\frac{1}{1-\delta E_{\omega} q(\omega)} E_{\omega}\left[q(\omega)(1-\delta) u_{i}(\omega)+\sum_{S} p(\omega, S) v_{i}(\omega, S)\right] .
$$

The reason $\phi^{2}$ is well-defined is precisely because $p$ is symmetric, so that the subscript $i$ on the right hand side of (2) no longer appears on the left hand side after integrating.

It is trivial to see that $\phi^{2}$ is continuous in $(p, q)$. Now compose the two correspondences, by defining a third correspondence $\phi: V \mapsto V$ :

$\phi(v)=\left\{v^{\prime} \in V \mid v^{\prime}=\phi^{2}(p, q)\right.$ for $(p, q)$ with $\left.(p(\omega), q(\omega)) \in \phi^{1}(\omega, v) \forall \omega\right\}$.

Since $\phi^{2}$ is a continuous function on a non-empty, covex and upper-hemi continuous correspondence (by Lemma 1$), \phi(v)$ is nonempty, convexvalued, and upper-hemi-continuous (uhc) in $v$. Hence, $\phi$ must have a fixed point, call it $v^{*}(n)$. Define an associated probability system $(p, q)$ by the particular value of $(p, q)$ in $(3)$ that permits the fixed point to be attained. One can now check that all the five conditions for an equilibrium are satisfied.

\section{Fragility of Mutual Help Groups}


Proposition 2. For any $\epsilon>0$, there exists $\bar{n}$ so that for all $n \geq \bar{n}$, $\operatorname{Frag}(n)>1-\epsilon$.

Proof. Assume that the proposition is false. Then there exists $\epsilon>0$ and an infinite set $N$ of group sizes such that for all $n \in N, \operatorname{Frag}(n) \leq 1-\epsilon$. By our assumptions on the cost function, there exists a closed interval $I(p)$ containing $p$ in its interior and a value $\underline{c}>0$ such that $c(q, \alpha) \geq \underline{c}$ for all cost shocks $\alpha$ and $q \in I(p)$. Fix this interval and the lower bound on costs in what follows.

Note that per-capita group payoffs are obviously bounded. It follows that for every $\mu>0$, there exists $n(\mu) \in N$ so that $v^{*}(n)-v^{*}(n(\mu))<\mu$ for all $n>n(\mu), n \in N$. Pick any such $\mu<\frac{1-\delta}{\delta} \underline{c}$, where $\underline{c}$ is described in the previous paragraph.

An application of the weak law of large numbers yields the following implication: For every $\epsilon>0$ there exists a group size $\hat{n}$ such that for every $n \in N$ with $n>\hat{n}$, the joint event that

(a) the number of actual donors exceeds $n(\mu)$, and

(b) the proportion of those in need lies within $I(p)$

has probability exceeding $1-\epsilon$. But in this event, we may use the definition of $\mu$ to conclude that for all individuals $i$ in some subgroup of size $n(\mu)$,

$$
c\left(q, \alpha_{i}\right) \geq \underline{c}>\frac{\delta}{1-\delta} \mu>\frac{\delta}{1-\delta}\left[v^{*}(n)-v^{*}(n(\mu))\right],
$$

so that (??) holds for $s=n(\mu)$. We may therefore conclude that the fragility of all such $n \in N$ exceeds $1-\epsilon$, which is a contradiction.

Proposition 3. For any $\mu>0$, there exists $\bar{\delta}<1$ and $\bar{n}$ so that, for all $n \geq \bar{n}$ and all $\delta \geq \bar{\delta}, \operatorname{Frag}(n)<\mu$.

Proof. Fix $\mu>0$. Now choose a size $m>1$ and a value $\epsilon$ with $\widetilde{v}(m)-\widetilde{v}(1)>\epsilon>0$, and define

$$
\bar{c}(\delta) \equiv \frac{\delta}{1-\delta}[\widetilde{v}(m)-\epsilon-\widetilde{v}(1)] .
$$


Denote as $I(c)$ the set of $q$ so that $c(q, \alpha) \geq c$ for any cost shock $\alpha$. It is easy to select $\bar{\delta}$ so that $\max _{\alpha} c(p, \alpha)<\bar{c} \equiv \bar{c}(\bar{\delta})$.

The probability that $q \in I(\bar{c})$ is an upper bound on the $i$-fragility of a group with a value $v$ of at least $\underline{v}=\widetilde{v}(m)-\epsilon$.

It follows that for any group of size $n$, if $\hat{v}(n) \geq \underline{v}$, a lower bound on the utility of the agents is given by

$$
v(n)=\frac{1}{[1-\delta \operatorname{Pr}(q \notin I(\bar{c}))]} \sum_{k=0}^{n-1} p(k, n-1)\left[p r\left(\frac{k+1}{n}\right) b-(1-p) d\left(\frac{k}{n}\right) \operatorname{Ec}\left(\frac{k}{n}, \alpha\right)\right] .
$$

An application of the weak law of large numbers yields the following implication: for every $\eta>0$ there exists a group size $\hat{n}(\eta)$ such that for every $n>\hat{n}(\eta)$, the probability that the proportion of those in need lies within $I(\bar{c})$ has probability less than $\eta$. It follows that we can choose $\eta$ sufficiently small that (i) $v(n) \geq \underline{v}$ for all $n>\hat{n}(\eta$ ), and (ii) $\eta<\mu$.

Since $\bar{c}(\delta)$ is increasing, it follows that, for $\delta>\bar{\delta}$, the fragility of all groups of size $n>\hat{n}(\eta)$ will be less than $\mu$.

\section{Social Networks.}

Proposition 4. For any graph $g \subset g^{c}, \delta\left(g^{c}\right) \leq \delta(g)$ and the inequality is strict for some $g$.

Proof. Let $w_{i}(g, \omega)$ denote the net payoff of $i$ at state $\omega$. For any $\epsilon$, let $\underline{p}$ be such that for all $i, \sum_{\omega \mid \omega \neq \omega_{r i}}$ for some i $w_{i}(g, \omega)<\epsilon$. Then,

$$
\begin{aligned}
\widetilde{v}_{j}(g)-\widetilde{v}_{i}(g) & >p b\left(\rho_{j}\left(g, \omega_{r j}\right)-\rho_{i}\left(\omega_{r i}\right)+p c\left(\sum_{k, i k \in g} \eta_{i}\left(g, \omega_{r k}\right)-\sum_{k, j k \in g} \eta_{j}\left(g, \omega_{r k}\right)\right)-\epsilon\right. \\
& >p c\left(\sum_{k, i k \in g} \eta_{i}\left(g, \omega_{r k}\right)-\sum_{k, j k \in g} \eta_{j}\left(g, \omega_{r k}\right)\right)-\epsilon .
\end{aligned}
$$

Hence, one can choose $\epsilon$ and hence $\underline{p}$ so that $\widetilde{v}_{j}(g)-\widetilde{v}_{i}(g)>0$.

By a similar argument, for any $\epsilon$, define $\bar{p}$ so that for all $i, i, \sum_{\omega \mid \omega \neq \omega_{d i}}$ for some $\mathrm{i} w_{i}(g, \omega)<$ $\epsilon$. Then, 


$$
\begin{aligned}
\widetilde{v}_{i}(g)-\widetilde{v}_{j}(g) & >(1-p) b\left(\sum_{k, i k \in g} \rho_{i}\left(g, \omega_{d k}\right)-\sum_{k, j k \in g} \rho_{j}\left(g, \omega_{d k}\right)\right)+(1-p) c\left(\eta_{j}\left(g, \omega_{d j}\right)-\eta_{i}\left(g, \omega_{d i}\right)\right. \\
& >(1-p) b\left(\sum_{k, i k \in g} \rho_{i}\left(g, \omega_{d k}\right)-\sum_{k, j k \in g} \rho_{j}\left(g, \omega_{d k}\right)\right)-\epsilon
\end{aligned}
$$

establishing the result.

Proof. We show that $\widetilde{v}_{i}\left(g^{c}\right) \geq \min _{i} \widetilde{v}_{i}(g)$ for any graph $g$, with strict inequality for some graphs $g$.

Notice that for any state $\omega$,

$\sum_{i \mid i=R} \rho_{i}(g, \omega)=\sum_{i \mid i=D} \eta_{i}(g, \omega) \leq \min \{k(\omega), n-k(\omega)\}=\sum_{i \mid i=R} \rho_{i}\left(g^{c}, \omega\right)=\sum_{i \mid i=D} \eta_{i}\left(g^{c}, \omega\right)$,

Hence,

$\sum_{i \mid i=R} \rho_{i}(g, \omega) b-\sum_{i \mid i=D} \eta_{i}(g, \omega) E c(\alpha) \leq \sum_{i \mid i=R} \rho_{i}\left(g^{c}, \omega\right) b-\sum_{i \mid i=D} \eta_{i}\left(g^{c}, \omega\right) E c(\alpha)$.

Taking expectations over $\omega$,

$$
\sum_{i} \widetilde{v}_{i}(g) \leq \sum_{i} \widetilde{v}_{i}\left(g^{c}\right)
$$

By anonymity, $\widetilde{v}_{i}\left(g^{c}\right)=\frac{\sum_{i} \widetilde{v}_{i}\left(g^{c}\right)}{n}$.

This shows that $\widetilde{v}_{i}\left(g^{c}\right) \geq \min _{i} \widetilde{v}_{i}(g)$ for any graph $g$. Furthermore, if the graph $g$ is not symmetric or disconnected, $\widetilde{v}_{i}\left(g^{c}\right)>\min _{i} \widetilde{v}_{i}(g)$.

Now consider $\bar{\delta}$ such that

$$
\frac{\bar{\delta}}{1-\bar{\delta}}\left(\widetilde{v}_{i}\left(g^{c}\right)-\widetilde{v}(1)\right)=\max _{\alpha} c(\alpha) .
$$

For any $\delta \geq \bar{\delta}, \operatorname{Frag}\left(g^{c}\right)=0$ and as $\widetilde{v}_{i}\left(g^{c}\right) \geq \min _{i} \widetilde{v}_{i}(g)$ for all $g$ with strict inequality for some $g$, the conclusion follows. 